\title{
Targeted therapeutic mild hypercapnia after cardiac arrest
}

\author{
Glenn M. Eastwood ${ }^{1,2^{*}}$, Alistair Nichol ${ }^{2,3,4}$ and Matt P. Wise \\ See related research by Sekhon et al., https:/ccforum.biomedcentral.com/articles/10.1186/s13054-017-1670-9
}

We read the paper entitled "Clinical pathophysiology of hypoxic ischemic brain injury after cardiac arrest: a "two-hit" model" by Sekhon et al. published in Critical Care [1]. We agree that the clinical pathophysiologic impact of hypoxic ischemic brain injury after cardiac arrest (CA) is multi-factorial and complex. We are concerned, however, that the description of carbon dioxide management fails to address the potential therapeutic role of mild hypercapnia and identify current research directions underway to explicitly investigate targeted mild hypercapnia in the early post-resuscitation period.

Previously, from a large retrospective audit involving $>16,000$ patients from 125 Australian and New Zealand (ANZ) intensive care units (ICUs) following CA between 2000 and 2011, we found that, compared with normocapnia, hypercapnia was associated with a greater likelihood of discharge home among survivors [2]. More recently, the findings of our prospective phase II multicentre randomised trial, the CCC trial, showed that targeting therapeutic mild hypercapnia (TTMH) $\left(\mathrm{PaCO}_{2}\right.$ 50-55 $\mathrm{mmHg}$ ), compared to targeted normocapnia (TN)
$\left(\mathrm{PaCO}_{2}\right.$ 35-45 mmHg) was feasible, appeared safe and resulted in attenuation of neuron specific enolase (NSE) release (a biomarker of brain injury) in resuscitated CA patients [3]. While at this stage only hypothesis generating, such findings suggest that mild hypercapnia could have neuro-protective properties during the immediate post-resuscitation phase. If proven to be beneficial, induction of hypercapnia would be an easy to apply intervention at minimal cost to most CA patients.

We agree that the current best evidence indicates that hypocapnia should be avoided, there is uncertainly whether normocapnia or TTMH is the optimal approach in the immediate post-resuscitation phase. There is sufficient uncertainty to justify a definitive trial to evaluate the potential benefit of mild hypercapnia. Indeed, we have now initiated the TAME Cardiac Arrest trial (Clinicaltrials.gov NCT03114033). This phase III multi-centre randomised controlled trial will determine whether TTMH, applied during the early post-resuscitation period, improves neurological outcome of resuscitated adult cardiac arrest patients admitted to the intensive care unit.

\section{Authors' response}

\section{Mypinder S. Sekhon, Philip N. Ainslie and Donald E. Griesdale}

\section{To the editor:}

We acknowledge Eastwood et al. for their insightful comments to our narrative review entitled "Clinical pathophysiology of hypoxic ischemic brain injury after cardiac arrest: a "two hit" model". They identify the importance of preventing secondary cerebral injury after cardiac arrest and the crucial role of arterial carbon dioxide induced modulation of cerebral blood

\footnotetext{
* Correspondence: glenn.eastwood@austin.org.au

${ }^{1}$ Department of Intensive Care, Austin Hospital, Melbourne, Victoria, Australia

${ }^{2}$ Australia and New Zealand Intensive Care Research Centre, Monash

University, Melbourne, Victoria, Australia

Full list of author information is available at the end of the article
}

flow and oxygen delivery. This effect occurs through the modulation of extracellular $\mathrm{pH}$ and is rendered less effective over time. Clearly, episodes of hypocapnia are associated with adverse outcome, stemming from cerebral vasoconstriction, reduced cerebral blood flow and oxygen delivery [1]. Conversely, in a large multicenter observational study, Schneider et. al demonstrated that patients with hypercapnia $(\mathrm{PaCO} 2>$ $45 \mathrm{mmHg}$ on one blood gas in the first 24 hours) had higher rates of discharge home among survivors (OR 1.16, 95\%CI: 1.03 - 1.32) [2]. 
Biologically, the phenomenon of "no reflow", characterized by cerebral vasoconstriction, microvascular thrombi and subsequent cerebral oligemia early after resuscitation is well established [1]. It follows that mild hypercapnia to induce cerebral vasodilation may augment blood flow during this critical period and mitigate secondary injury. We commend Eastwood et al. for undertaking a foundational randomized control trial to investigate the role of mild hypercapnia $\left(\mathrm{PaCO}_{2}\right.$ 50-55 $\mathrm{mmHg}$ ) after cardiac arrest compared with normocapnia $\left(\mathrm{PaCO}_{2}\right.$ 35-45 mmHg) (TAME trial; Clinicaltrials.gov NCT03114033) and eagerly await the results.

This trial will no doubt provide important insights into the management of post-resuscitative care of cardiac arrest patients. However, it should be noted that there is likely significant within-patient heterogeneity with respect to individual pathophysiology of hypoxic ischemic brain injury [1]. Randomized control trials of single physiological interventions in critical care may fail to account for these nuances. For example, two recent randomized trials of single physiological interventions aimed at mitigating secondary injury after cardiac arrest and traumatic brain injury, specifically targeted temperature management and transfusion thresholds, respectively, were negative $[4,5]$. Hence our belief is that we should shift the paradigm to delineating the underlying individualized pathophysiology and establish personalized physiologic resuscitation targets following cardiac arrest. Our research group has recently demonstrated the ability to monitor cerebral autoregulation in real time after cardiac arrest to identify optimal and individualized mean arterial pressure [6]. We acknowledge that it remains unknown if individualized perfusion targets are associated with improved outcome after cardiac arrest. However, this strategy represents an intriguing shift towards personalized physiological resuscitation in the management of this catastrophic and complex disease.

\footnotetext{
Abbreviations

CA: Cardiac arrest; TN: Targeted normocapnia; TTMH: Targeted therapeutic mild hypercapnia
}

\section{Acknowledgements}

Not applicable.

Funding

No funding source.

Availability of data and materials

Not applicable.

\section{Authors' contributions}

GE, AN and MW wrote and edited the letter and all authors read and approved the final manuscript.

Ethics approval and consent to participate Not applicable.
Consent for publication

Not applicable.

\section{Competing interests}

The authors declare that they have no competing interests.

\section{Publisher's Note}

Springer Nature remains neutral with regard to jurisdictional claims in published maps and institutional affiliations.

\section{Author details}

${ }^{1}$ Department of Intensive Care, Austin Hospital, Melbourne, Victoria, Australia. ${ }^{2}$ Australia and New Zealand Intensive Care Research Centre, Monash University, Melbourne, Victoria, Australia. ${ }^{3}$ Department of Intensive Care, Alfred Hospital, Melbourne, Victoria, Australia. ${ }^{4}$ rish Critical Care- Clinical Research Network, University College Dublin / St Vincent's University Hospital, Dublin, Ireland. ${ }^{5}$ Adult Critical Care, University Hospital of Wales, Cardiff, UK.

Published online: 31 July 2017

References

1. Sekhon MS, Ainslie PN, Griesdale DE. Clinical pathophysiology of hypoxic ischemic brain injury after cardiac arrest: a "two-hit" model. Crit Care. 2017; 21:90.

2. Schneider AG, Eastwood GM, Bellomo R, et al. Arterial carbon dioxide tension and outcome in patients admitted to the intensive care unit after cardiac arrest. Resuscitation. 2013;84:927-34.

3. Eastwood GM, Schneider AG, Suzuki S, et al. Targeted therapeutic mild hypercapnia after cardiac arrest: A phase II multi-centre randomised controlled trial (the CCC trial). Resuscitation. 2016;104:83-90.

4. Nielsen N, Wetterslev J, Cronberg T, Erlinge D, Gasche Y, Hassager C, et al. Targeted temperature management at $33{ }^{\circ} \mathrm{C}$ versus $36{ }^{\circ} \mathrm{C}$ after cardiac arrest. N Engl J Med. 2013;369:2197-206.

5. Robertson CS, Hannay HJ, Yamal J-M, Gopinath S, Goodman JC, Tilley BC, et al. Effect of erythropoietin and transfusion threshold on neurological recovery after traumatic brain injury: a randomized clinical trial. JAMA. 2014; 312:36-47.

6. Sekhon MS, Smielewski P, Bhate TD, Brasher PM, Foster D, Menon DK, et al. Using the relationship between brain tissue regional saturation of oxygen and mean arterial pressure to determine the optimal mean arterial pressure in patients following cardiac arrest: A pilot proof-of-concept study. Resuscitation. 2016;106:120-5. 